\title{
Opinion
}

\section{Paediatric Virology and innovation in medical education: An interview with Dr Ioannis N. Mammas, Consultant Paediatrician on the island of Euboea (Greece) and Coordinator of the Paediatric Virology Study Group}

\author{
DEMETRIOS A. SPANDIDOS
}

Laboratory of Clinical Virology, School of Medicine, University of Crete, 71003 Heraklion, Greece

Received July 22, 2019; Accepted August 26, 2019

DOI: $10.3892 /$ etm.2019.7950

\begin{abstract}
Born in Chalkida in Greece, Dr Ioannis N. Mammas, Consultant Paediatrician on the island of Euboea, studied Medicine at the University of Crete School of Medicine, where he received his MD in 2002 and his $\mathrm{PhD}$ in Clinical Virology in 2006. He was trained in Paediatrics and Neonatology in Merseyside (UK), attending the 'Alder Hey' Children's Hospital's specialty training (ST) rotation programme and in Athens (Greece) at the 'Penteli' Children's Hospital and the 'MAKKA' Paediatric Infectious Diseases (PID) Unit of the 'Aghia Sophia' Children's Hospital. On October 10th, 2015 , he presented the Paediatric Virology subspecialty's proposal, which up-to-date has attracted the critical interest of several international experts in the scientific fields of Neonatology, Paediatrics, PID and Virology and has been evaluated as a unique project of innovation in medical education. According to Dr Mammas, Paediatrics is the specialty, which daily provides the opportunity to support with responsibility the children's smile, the hope of life and future. He highlights the significant role of the Paediatric Virology Study Group (PVSG), a paediatric think tank, which was established by a group of paediatric trainees and young paediatricians in 2007 and since then has been involved with enthusiasm in the perspectives of Paediatric Virology in medical education. Dr Mammas strongly believes that in the future, Paediatric Virology subspecialists will have a strategically principal role, both clinical and academic, at the fight against viral infections in childhood. This aim will be supported by the newly founded Institute of Paediatric Virology (IPV), the first scientific initia-
\end{abstract}

Correspondence to: Professor Demetrios A. Spandidos, Laboratory of Clinical Virology, School of Medicine, University of Crete, 71003 Heraklion, Greece

E-mail: spandidos@spandidos.gr

Key words: Paediatric Virology, Paediatric Virology Study Group, Institute of Paediatric Virology, Euboea, Greece, Ioannis N. Mammas tive in the world committed to medical education on neonatal and paediatric viral infections.

\section{Contents}

1. Introduction

2. Questions and Answers

\section{Introduction}

Born in Chalkida, Greece, in 1978, Dr Ioannis N. Mammas (Fig. 1) spent his childhood in Aliveri on the island of Euboea. He studied Medicine at the University of Crete School of Medicine, and he received his MD with honours in 2002. During the academic year 2001/2002, he received a Boston University research scholarship and visited the Boston University School of Medicine in Boston, USA. He continued his postgraduate studies on human papillomaviruses (HPV) at the Department of Clinical Virology of the University of Crete School of Medicine, where in 2006, he obtained his PhD (Final Diploma mark: Excellent) in Clinical Virology (1-6).

After the fulfilment of his military service, he moved to the UK, where he attended the specialty training (ST) rotation programme of the 'Alder Hey' Children's Hospital in Paediatrics and Neonatology. He worked at the neonatal and paediatric wards of the Wirral University Teaching Hospital, the Southport and Ormskirk Hospital, the Noble's Hospital and the Countess of Chester Hospital NHS Trust, specializing in Neonatology, Paediatrics and Paediatric Accident \& Emergency (A\&E) Medicine. In July 2008, he attended the Paediatric Intensive Care Unit (PICU) of the 'Alder Hey' Children's Hospital in Liverpool under the direction of Dr Jane Ratcliffe, Consultant Paediatric Intensivist. Following his return to Greece, he worked at the First Department of Paediatrics at the 'Penteli' Children's Hospital in Athens under the direction of Dr Nikolaos Myriokefalitakis. He also attended the 'MAKKA' Paediatric Infectious Diseases (PID) Unit of the 'Aghia Sophia' Children's Hospital in Athens under the supervision of Professor Maria Theodoridou, Professor of Paediatrics at the University of Athens School of Medicine. 
He has published more than 90 peer-reviewed articles in international and Hellenic academic journals on Paediatrics, Virology, Infectious Diseases, Neonatology and Preventive Medicine. His academic and research interests focus on Paediatric Virology, HPV, viral infections in infancy and childhood, public health and medical education. Since 2007, he is a Coordinator of the Paediatric Virology Study Group (PVSG) (7) and since 2015, Chair of the organizing committee of the 'workshops on Paediatric Virology' (8-13).

On October 10th, 2015, he presented the '2015 Paediatric Virology proposal' (14), which, to date, has attracted the critical interest of several leading experts in the scientific fields of Neonatology, Paediatrics, PID and Virology (15). Although this debate has already tagged difficulties, challenges and limitations on the role of Paediatric Virology as a separate paediatric subspecialty, the '2015 Paediatric Virology proposal' has been evaluated as 'a call for innovation in paediatric education' (16) and 'an educational goal for advanced academic excellence' (17); the potential value of Paediatric Virology subspecialists in the future has undoubtedly been accepted (16-28). Currently, Dr Mammas is a Consultant Paediatrician on the island of Euboea in Greece, Research Collaborator at the Department of Clinical Virology of the University of Crete School of Medicine and honorary Research Fellow at the First Paediatric Department of the University of Athens School of Medicine, which he joined in 2010. Recently, he co-founded the Institute of Paediatric Virology (IPV), the first international scientific initiative in the world, which is committed to medical education on the scientific field of Paediatric Virology (Fig. 2). The IPV is based on the island of Euboea (Greece), is supported by the Department of Clinical Virology of the University of Crete School of Medicine and the First Department of Paediatrics of the University of Athens School of Medicine and its establishment is under the auspices of the World Academy of Sciences (WAS).

\section{Questions and Answers}

Question: What is the Paediatric Virology Study Group (PVSG)?

Answer: The PVSG is a think tank, which consists of a group of paediatric trainees and young paediatricians, which aims to promote the new scientific field of Paediatric Virology as a new paediatric subspecialty. It was established in 2007 in Liverpool at the Merseyside in the UK, where I worked as a Senior House Officer in Neonatology at the Wirral University Teaching Hospital. This effort was devoted to two twin neonates with congenital cytomegalovirus (CMV) infection, who were treated in our local Neonatal Unit and required the collaboration of several specialists in Neonatology, PID and Microbiology. At that time, the necessity of good knowledge on Clinical Virology emerged and the value of specialization in the field of Paediatric Virology was evaluated positively by all members of our medical team.

Question: During all these 12 years of the PVSG, you have been involved with enthusiasm in the perspectives of Paediatric Virology in medical education. What is Paediatric Virology? Answer: Paediatric Virology is the branch of Paediatrics, which deals with the study of the pathophysiology, the diag-

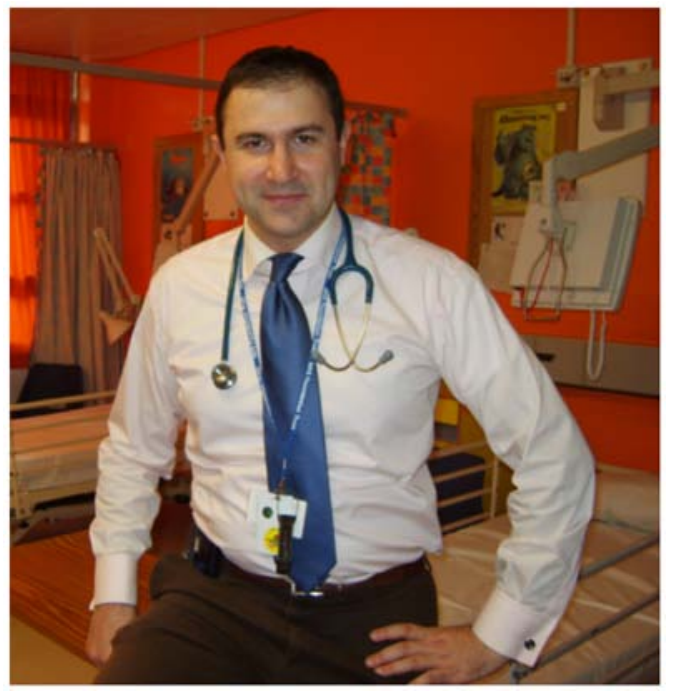

Figure 1. Dr Ioannis N. Mammas, MD, PhD, Consultant Paediatrician on the island of Euboea (Greece), Coordinator of the Paediatric Virology Study Group (PVSG) and Co-founder of the Institute of Paediatric Virology (IPV).

nostic procedures, the prevention strategies and the treatment options of viral infections in newborns, children and adolescents. During the previous decades, Paediatric Virology was not considered as an isolated discipline and was included in the Paediatric Infectious Diseases section within Paediatrics. However, recent advances on the field of Clinical Virology, using new diagnostic methods and the increasing needs of specific patient groups, such as preterm neonates, hospitalized children at the PICU, HIV-infected or transplanted children, have made this scientific field distinct and highly evolving, both at undergraduate and postgraduate level. In the future, paediatric virologists are expected to have a strategically principal role, both clinical and academic, at the fight against viral infections in childhood.

Question: Can Paediatric Virology be implemented into clinical practice as an independent paediatric subspecialty?

Answer: The PVSG, as well as several leading experts in the field of Neonatology, Paediatrics and Virology believe that it can. However, the implementation of this proposal into clinical practice, as first formulated and presented in 2015 in Athens during the '1st workshop on Paediatric Virology', remains the subject for further scientific and academic evaluation. It is important to determine carefully how paediatric virologists can help paediatric clinical practice especially during the job limitation era and the understandable fears encountered by subspecialisation. This definitely needs more time, passion and inspiration.

Question: You have been the Chair of the organizing committee of all the 'workshops on Paediatric Virology' during the period 2015-2019. What was the outcome of these workshops?

Answer: The 'workshops on Paediatric Virology', organized by the PVSG, tried to promote international scientific exchange and cooperation of both clinicians and researchers on Paediatric Virology. During these workshops, we also had the chance to discuss recent advances on neonatal and paediatric viral infections, as well as several new ideas in our field, such 


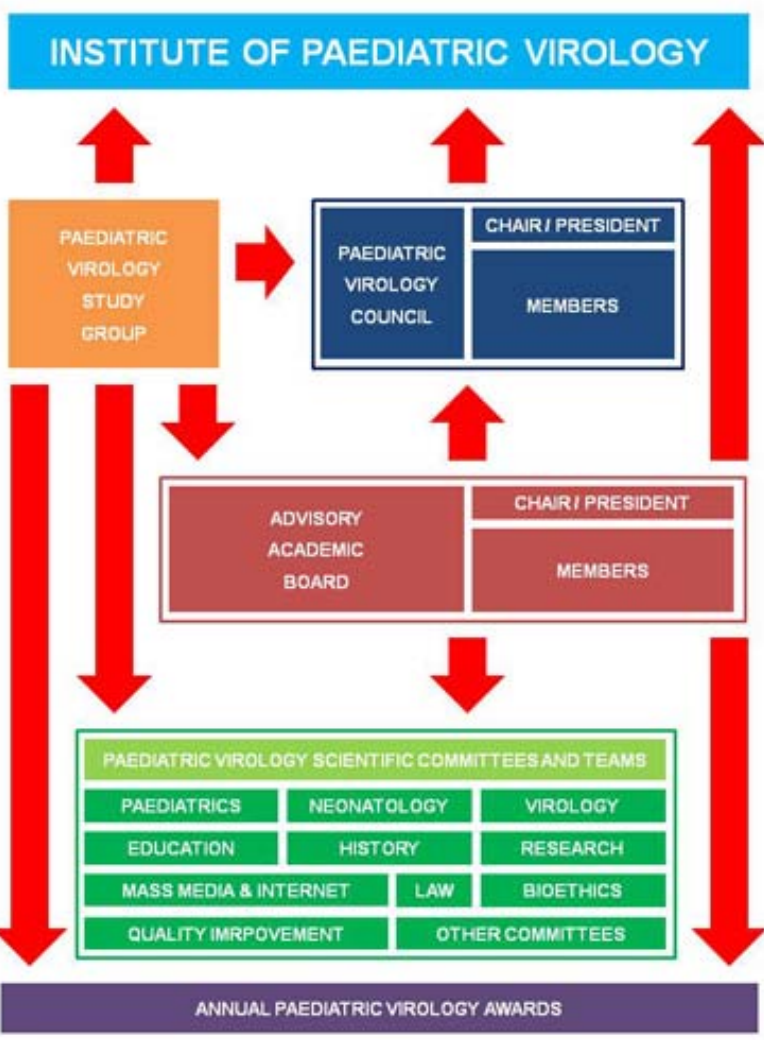

Figure 2. The structure of the newly founded Institute of Paediatric Virology (IPV) based on the island of Euboea.

as the implementation of new technologies in the diagnosis and management of viral infections, the structure of the newly founded Institute of Paediatric Virology (IPV), as well as the necessity of the establishment of the European Society for Paediatric Virology (ESPV). To date, the PVSG has provided the key points of all presentations from all meetings published in PubMed at the Experimental and Therapeutic Medicine and the International Journal of Molecular Medicine, with free open access.

Question: How did you choose to be trained in Paediatrics? Answer: I was attending as a medical student Paediatric Intensive Medicine in Crete and that day I had stayed late studying case-reports at the doctor's meeting room of the local PICU. An unexpected phone call from south Crete informed us about a child, an 8-year-old boy with meningitis; the child was referred by the local Healthcare Centre to the University Hospital of Heraklion. When the boy arrived in PICU, he was unconscious and haemorrhagic rash had already appeared. Although it was impossible to save this child, the preparedness of our Unit, the protocol-based PICU approach and the team working of all medical and nursing staff was state-of-the-art. I was really fascinated by the science that I was due to choose, Paediatrics; I was also impressed by the quality of my future colleagues, the paediatric health providers, feeling really proud of them. It was not until the early hours of the next morning that the child eventually passed away because of meningococcal septicaemia. The type of microbe that was detected the next days in his cerebrospinal fluid and that had caused his death could have been prevented with the vaccina- tion against meningitis, which at that time had just started to be administered to all infants.

Question: In your last lecture in our workshop, you mentioned that you feel gratitude to your teachers in Paediatrics in the UK. So, England or 'Penteli' Children's Hospital in Greece? Where do you think that paediatric clinical training was more valuable for you?

Answer: Yes. I feel gratitude to all my British paediatric teachers and colleagues at the Wirral University Teaching Hospital, where the idea of Paediatric Virology was born in 2007, the Southport and Ormskirk Hospital, the Noble's Hospital, the Countess of Chester Hospital and the 'Alder Hey' Children's Hospital in Merseyside. In the UK, the level of training of young doctors in Paediatrics and Neonatology is exemplary, indeed. In Greece, I was fortunate to have as a teacher in Paediatrics one of the most talented Greek paediatric teachers in Athens, Dr Nikolaos Myriokefalitakis.

Question: Which was your most adventurous on-call in your career until now?

Answer: I was on-call with my Consultant and our Paediatric A\&E Assessment room was busy as always. It was 2:00 a.m., when a 4-year-old boy with a two-month history of paroxysmal cough attended A\&E with his father. In the past 4 weeks, the child had already been evaluated three times and his prescribed inhalers two weeks ago had made no difference in his coughing. 'I am really sorry, doctor, that we attended the hospital so late, but I really worry about my child' the father explained to us that his child's case was an emergency. And it was an emergency, indeed. The saturation monitoring showed less than $92 \%$. Chest auscultation revealed that there was no air entry at his right lung. We asked for a chest X-ray, which showed right-sided pneumothorax. The child was referred to our paediatric surgical team, who immediately managed the pneumothorax. The new X-ray showed a mass at the right lung, and the child was referred for computed tomography (CT) imaging, which proposed the diagnosis of pulmonary blastoma, an extremely rare neoplasm in childhood. The boy was operated by a thoracic surgeon in one of the private Hospitals in Greece and now he is doing well.

Question: Since 2010, you are a Consultant Paediatrician on the island of Euboea in Greece. How is your paediatric experience in the community? How this experience is related to Paediatric Virology?

Answer: Paediatrics is the specialty, which daily provides the opportunity to support the children's smile, the hope of life and future. This responsibility is great because our science supports what is most valuable in our society, the lives and health of children. Practicing Paediatrics on my island makes this responsibility even greater. Regarding your second question, I have to note that $>90 \%$ of our A\&E attendances at the general paediatric clinic are children with a viral infection. To date, the diagnostic and therapeutic management of all these children has been an excellent school on Paediatric Virology! A school, though, which everyday highlights that there is so much to be done in our field, in our clinical practice, in research, as well as in medical education. 
Question: The island of Euboea is the island of Dr George N.Papanicolaou. How do you feel that you continue the medical tradition of your island of Dr George N. Papanicolaou?

Answer: For us, the young scientists on the island of Euboea, Dr George N. Papanicolaou is an outstanding figure of respect and inspiration. The scientists, though, who are continuing his contribution in Medicine are located elsewhere, in the top leading research centres, worldwide, where research continues to give hope and pulse to the future of humanity.

Question: In 2014, you met Nobelist Laureate Professor Harald zur Hausen in Heidelberg (Germany). How significant was that meeting for your scientific attempt on Paediatric Virology?

Answer: As a junior paediatrician, who has had completed a $\mathrm{PhD}$ on HPV, this was a really outstanding honour! One year after this meeting, the PVSG created the 'workshop on Paediatric Virology', while three years later, we had the great honour of Professor Harald zur Hausen to be included to the chairpersons of our workshop. I remember his first words, when he reported with enthusiasm that he had attended ancient Greek lessons during his highschool studies. 'There is a very beautiful Greek word that you use to describe freedom: El-ef-the-ri-a! Eleftheria! This is the most beautiful Greek word, and it is an ancient Greek word' he had told me. 'Elfetheria?' I was impressed by the accurate way that he was spelling this word. 'Yes, eleftheria! And you, the Greeks, you have a unique way to support it', he said.

Question: Is the discovery of HPV causing cervical cancer or the discovery of the vaccines against HPV a more important medical discovery?

Answer: Undoubtedly, both discoveries were very powerful moments of modern Medicine. The Nobel Prize was given to the scientist who discovered in the mid-1970s, the causative relationship between HPV and cervical carcinogenesis, while the implementation of the vaccination against HPV into clinical practice is one of the most important medical challenges of our last decade.

Question: But why do the coverage rates against HPV among female adolescents in Greece remain low? How could be this changed?

Answer: A combination of lack of information and the outcome of the recent Greek economic crisis. It is, however, quite interesting that our scientific community has difficulty in understanding the obstacles that clinical professionals face when they try to apply a new scientific discovery into clinical practice. It remains for us, the community-based paediatricians, to do our best to apply modern medical achievements to the children's population in our country. What we need is medical education, inspiration, optimism and passion! As young scientists, we feel that we have all of them and we will make it!

Question: Recently, you co-founded the IPV, the first international scientific initiative in the world, which is committed to medical education on the new, bold, scientific field of Paediatric Virology, under the auspices of the World Academy of Sciences (WAS) and the support of the Department of Clinical Virology of the University of Crete School of Medicine and the First Department of Paediatrics of the University of Athens School of Medicine. What will be the priorities of the IPV in the near future?

Answer: We will focus on three major directions. The first one is to provide an educational e-platform on the current clinical practice and recent research advances on the prevention, diagnosis, management and therapeutic strategies against neonatal and paediatric viral infections. The second one is to continue to facilitate scientific discussion and exchange between virologists and paediatric health professionals and to encourage them to work together as an international network to achieve the promotion of paediatric health towards viral infectious diseases. The third and most important direction is the planning, evaluation and implementation into the clinical practice of a new module of Paediatric Virology subspecialisation for paediatric trainees and junior health professionals. This programme will be conducted in collaboration with worldwide state-of-the-art training centres, both clinical and laboratory-based, on neonatal and paediatric viral infections. Our aim is to add a tiny 'mosaic tile' in future Paediatrics, the most beautiful specialty in Medicine.

Question: Thank you. I would like to congratulate you for your innovative proposal in Paediatric Virology as well as for your significant contribution in the foundation of the IPV. You have all of my support and I will be always available for any requested consultation and contribution.

\section{Acknowledgements}

This article is published in the third supplement issue of the Experimental and Therapeutic Medicine, which is dedicated to Paediatric Virology. This edition is performed in the context of the '5th workshop on Paediatric Virology' (Sparta, Greece, October 12, 2019) organized by the Paediatric Virology Study Group (PVSG) and supported by the Department of Clinical Virology of the University of Crete School of Medicine and the First Department of Paediatrics of the University of Athens School of Medicine. I would like to thank Dr Ioannis N. Mammas for this educational and inspirational interview-style article. I would also like to thank all the members of the PVSG for their interesting questions to Dr Mammas and their valuable comments.

\section{References}

1. Mammas IN: Human papilloma virus HPV in adults and children (PhD Thesis). University of Crete School of Medicine, Heraklion, 2006.

2. Mammas IN, Sourvinos G, Michael C and Spandidos DA: Human papilloma virus in hyperplastic tonsillar and adenoid tissues in children. Pediatr Infect Dis J 25: 1158-1162, 2006.

3. Mammas I, Sourvinos G, Michael C and Spandidos DA: High-risk human papilloma viruses (HPVs) were not detected in the benign skin lesions of a small number of children. Acta Paediatr 97: 1669-1671, 2008.

4. Mammas IN, Sourvinos G and Spandidos DA: Human papilloma virus (HPV) infection in children and adolescents. Eur J Pediatr 168: 267-273, 2009.

5. Mammas IN, Sourvinos G, Vakonaki E, Giamarelou P, Michael C and Spandidos DA: Novel human papilloma virus (HPV) genotypes in children with recurrent respiratory papillomatosis. Eur J Pediatr 169: 1017-1021, 2010. 
6. Mammas IN, Sourvinos G and Spandidos DA: The paediatric story of human papillomavirus (Review). Oncol Lett 8: 502-506, 2014.

7. Mammas IN, Theodoridou $M$ and Spandidos DA: The development of the Paediatric Virology Study Group: Ten years in the making. Exp Ther Med 13: 363, 2017.

8. Mammas IN, Greenough A, Theodoridou M, Kramvis A, Christaki I, Koutsaftiki C, Koutsaki M, Portaliou DM, Kostagianni G, Panagopoulou P, et al: Current views and advances on Paediatric Virology: An update for paediatric trainees. Exp Ther Med 11: 6-14, 2016.

9. Mammas IN, Theodoridou M, Kramvis A, Thiagarajan P, Gardner S, Papaioannou G, Melidou A, Koutsaki M, Kostagianni G, Achtsidis V, et al: Paediatric Virology: A rapidly increasing educational challenge. Exp Ther Med 13: 364-377, 2017.

10. Mammas IN and Spandidos DA: Athens-based meeting to discuss the paediatric virology crossroad in October 2017. Acta Paediatr 106: 1536, 2017.

11. Mammas IN, Greenough A, Theodoridou M, Kramvis A, Rusan M, Melidou A, Korovessi P, Papaioannou G, Papatheodoropoulou A, Koutsaftiki C, et al: Paediatric Virology and its interaction between basic science and clinical practice (Review). Int J Mol Med 41: 1165-1176, 2018

12. Mammas IN, Theodoridou M, Thiagarajan $P$ Melidou A, Papaioannou G, Korovessi P, Koutsaftiki C, Papatheodoropoulou A, Calachanis M, Dalianis T, et al: A paediatric influenza update 100 years after the Skyros island Spanish flu outbreak. Exp Ther Med 17: 4327-4336, 2019.

13. Mammas IN, Dalianis T, Doukas SG, Zaravinos A, Achtsidis V, Thiagarajan P, Theodoridou M and Spandidos DA: Paediatric virology and human papillomaviruses: An update. Exp Ther Med 17: 4337-4343, 2019.

14. Mammas IN, Greenough A, Theodoridou M and Spandidos DA: Paediatric Virology: A new paediatric subspecialty? A proposal at the Workshop on Paediatric Virology, Athens, October 10, 2015. Exp Ther Med 11: 3-5, 2016.

15. Mammas IN and Spandidos DA: The subspecialty of Paediatric Virology: A 'mosaic tile' in future Paediatrics. Exp Ther Med 12: 539-540, 2016

16. Mammas IN and Spandidos DA: Advancing challenges in Paediatric Virology: An interview with Professor Barbara A. Rath, Co-founder and Chair of the Vienna Vaccine Safety Initiative. Exp Ther Med 18: 3231-3237, 2019.

17. Mammas IN: Clinical Virology research and medical education in Greece: An interview with Professor Demetrios A. Spandidos, Professor of Clinical Virology at the University of Crete in Greece. Exp Ther Med 18: 3221-3225, 2019.
18. Mammas IN and Spandidos DA: Paediatric Virology and respiratory syncytial virus: An interview with Honorary Senior Lecturer in Paediatric Infectious Diseases Dr Simon B. Drysdale (St. George's, University of London, UK). Exp Ther Med 18: 3226-3230, 2019

19. Mammas IN and Spandidos DA: The educational challenge of Paediatric Virology: An interview with Professor of Neonatology Anne Greenough. Exp Ther Med 14: 3332-3334, 2017.

20. Mammas IN and Spandidos DA: Paediatric Virology as a new educational initiative: An interview with Nobelist Professor of Virology Harald zur Hausen. Exp Ther Med 14: 3329-3331, 2017.

21. Mammas IN and Spandidos DA: The proposal of Paediatric Virology and its perspectives: An interview with Professor of Paediatrics Maria Theodoridou. Exp Ther Med 14: 3338-3340, 2017.

22. Mammas IN and Spandidos DA: Evaluating the proposal of paediatric virology: An interview with Professor Tina Dalianis, Professor of Tumour Virology at Karolinska Institutet. Exp Ther Med 16: 2785-2789, 2018.

23. Mammas IN and Spandidos DA: The innovation of the subspecialty of Paediatric Virology: An interview with Research Professor of Molecular Virology Anna Kramvis. Exp Ther Med 14: 3335-3337, 2017

24. Mammas IN and Spandidos DA: Paediatric virology as a candidate of paediatric subspecialisation: An interview with Assistant Professor of Molecular Microbiology-Virology Angeliki Melidou. Exp Ther Med 16: 2795-2798, 2018.

25. Mammas IN and Spandidos DA: The future of medical education in neonatology, paediatrics and paediatric virology: An interview with Professor Alan Michael Weindling, Professor of Perinatal Medicine at the University of Liverpool. Exp Ther Med 16: 2805-2808, 2018.

26. Mammas IN and Spandidos DA: Neonatology, paediatrics and paediatric virology on a British island: An interview with neonatologist Dr Prakash Thiagarajan (Isle of Man). Exp Ther Med 16: 2790-2794, 2018.

27. Mammas IN and Spandidos DA: The philosophy of paediatric teaching: An interview with Dr Nikolaos Myriokefalitakis, former Clinical Director of the 'Penteli' Children's Hospital in Athens (Greece). Exp Ther Med 16: 2799-2802, 2018.

28. Mammas IN, Theodoridou M and Spandidos DA: The wisdom and eudaimonia of Paediatrics: An interview with Professor George P. Chrousos, Professor of Paediatrics and Endocrinology at the University of Athens, Greece. Exp Ther Med 18: 3217-3220, 2019.

This work is licensed under a Creative Commons Attribution-NonCommercial-NoDerivatives 4.0 International (CC BY-NC-ND 4.0) License. 